\title{
Spheromak formation and sustainment studies at the sustained spheromak physics experiment using high-speed imaging and magnetic diagnostics
}

\author{
C. A. Romero-Talamás a) \\ Applied Physics, California Institute of Technology, Pasadena, California 91125 \\ C. Holcomb \\ Lawrence Livermore National Laboratory, 7000 East Avenue, Livermore, California 94550 \\ P. M. Bellan \\ Applied Physics, California Institute of Technology, Pasadena, California 91125 \\ D. N. Hill \\ Lawrence Livermore National Laboratory, 7000 East Avenue, Livermore, California 94550
}

(Received 7 September 2005; accepted 3 November 2005; published online 8 February 2006)

\begin{abstract}
A high-speed imaging system with shutter speeds as fast as $2 \mathrm{~ns}$ and double frame capability has been used to directly image the formation and evolution of the sustained spheromak physics experiment (SSPX) [E. B. Hooper et al., Nucl. Fusion 39, 863 (1999)]. Reproducible plasma features have been identified with this diagnostic and divided into three groups, according to the stage in the discharge at which they occur: (i) breakdown and ejection, (ii) sustainment, and (iii) decay. During the first stage, plasma descends into the flux conserver shortly after breakdown and a transient plasma column is formed. The column then rapidly bends and simultaneously becomes too dim to photograph a few microseconds after formation. It is conjectured here that this rapid bending precedes the transfer of toroidal to poloidal flux. During sustainment, a stable plasma column different from the transient one is observed. It has been possible to measure the column diameter and compare it to CORSICA [A. Tarditi et al., Contrib. Plasma Phys. 36, 132 (1996)], a magnetohydrodynamic equilibrium reconstruction code which showed good agreement with the measurements. Elongation and velocity measurements were made of cathode patterns also seen during this stage, possibly caused by pressure gradients or $\mathbf{E} \times \mathbf{B}$ drifts. The patterns elongate in a toroidal-only direction which depends on the magnetic-field polarity. During the decay stage the column diameter expands as the current ramps down, until it eventually dissolves into filaments. With the use of magnetic probes inserted in the gun region, an X point which moved axially depending on current level and toroidal mode number was observed in all the stages of the SSPX plasma discharge. () 2006 American Institute of Physics. [DOI: 10.1063/1.2140682]
\end{abstract}

\section{INTRODUCTION}

The sustained spheromak physics experiment ${ }^{1}$ (SSPX) routinely produces spheromaks from a coaxial magnetized plasma source. The aim of these experiments is to understand how currents in the plasma produce the spheromak and the efficiency with which spheromak fields contain hot plasmas. A diverse set of diagnostics has been implemented to measure the spheromak internal density, temperature, and magnetic field. ${ }^{2-4}$ Many diagnostics have access to the plasma through a midplane opening of the flux conserver, as shown in Fig. 1. Recently, a high-speed imaging system was added to the SSPX diagnostic list.

Imaging as a diagnostic has the advantage of being completely passive and remote (i.e., does not perturb the plasma) yielding data with temporal and spatial resolutions. However,

\footnotetext{
a) Present address: Lawrence Livermore National Laboratory, 7000 East Avenue, Livermore, California 94550. Electronic mail: romerotalamas1@1ln1.gov
}

photographing spheromak plasmas is a challenging task because of the speed at which plasma features move, the short duration of the experiments (typically between microseconds to milliseconds), and the small amount of light when the plasma is highly ionized. For this reason, specially designed optical, vacuum, and electronic hardwares have been used to obtain plasma images. We have used these images to identify reproducible features that cannot be inferred by any other SSPX diagnostic. These features, which include evolving cathode patterns and a central plasma column, can be divided into three groups according to the stage of the discharge at which they occur, namely, (i) breakdown and ejection, (ii) sustainment, and (iii) decay. These stages are indicated on the typical gun current time profile shown in Fig. 2.

During the first stage, plasma is ejected from the gun describing what is inferred from the images as a helical expansion (i.e., traveling along and rotating around the axis of the chamber) filling up the flux conserver. The magnetic helicity, ${ }^{5,6} K=\int \mathbf{A} \cdot \mathbf{B} d^{3} r$, quickly builds up at this stage and is sustained against resistive losses as prescribed by 


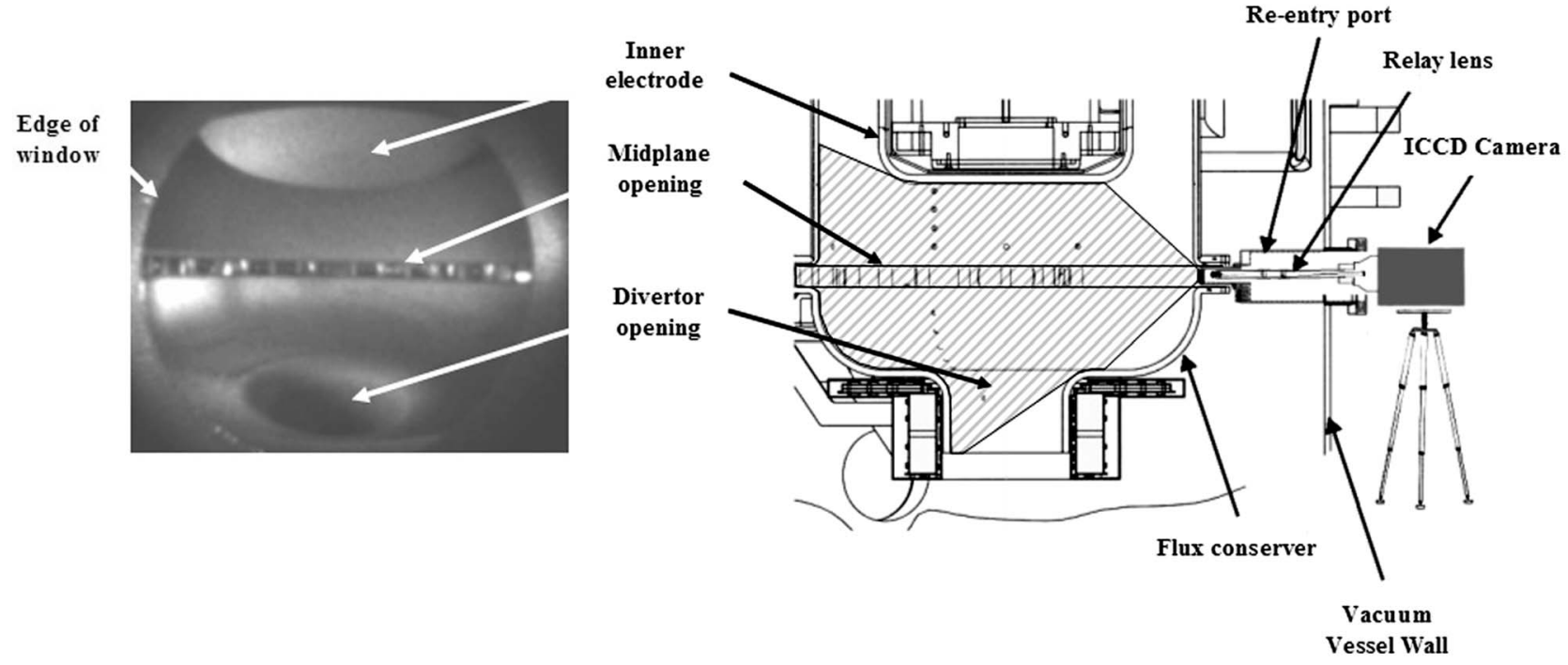

FIG. 1. Interior view of the SSPX flux conserver. A relay lens is used to convey the image from a wide angle lens to the ICCD camera. The view of the flux conserver through the relay lens is shown on the left image. The horizontal field of view is almost $1 \mathrm{~m}$.

$$
\frac{d K}{d t}=2 V_{g} \Psi_{g}-2 \int \eta \mathbf{J} \cdot \mathbf{B} d^{3} r
$$

where $V_{g}$ and $\Psi_{g}$ are gun voltage and flux, respectively, and $\eta$ is the plasma resistivity. Voltage spikes during the breakdown and ejection stage have been observed and coincide with images that indicate changes in just a few microseconds in a short-lived plasma column that appears during this early stage. These fast changes are conjectured to precede magnetic reconnection, which is a mechanism inherent to the formation of closed magnetic surfaces in spheromaks.

Edge probes presently installed in several locations of the SSPX flux conserver provide some insight into the plas- ma's magnetic evolution. However, with these probes it is not possible to resolve magnetic reconnection events. Equilibrium magnetohydrodynamic (MHD) solutions suggest the presence of X points in the SSPX gun region. For this reason, three arrays of magnetic probes were inserted in the gun in order to study reconnection in this region. These arrays showed the presence of an X point that appeared shortly after plasma breakdown, during sustainment, and during decay.

The organization of this paper is as follows. The experimental setup is presented in Sec. II. The breakdown and ejection images and magnetic probe data are presented in Sec. III. The sustainment stage, including cathode pattern

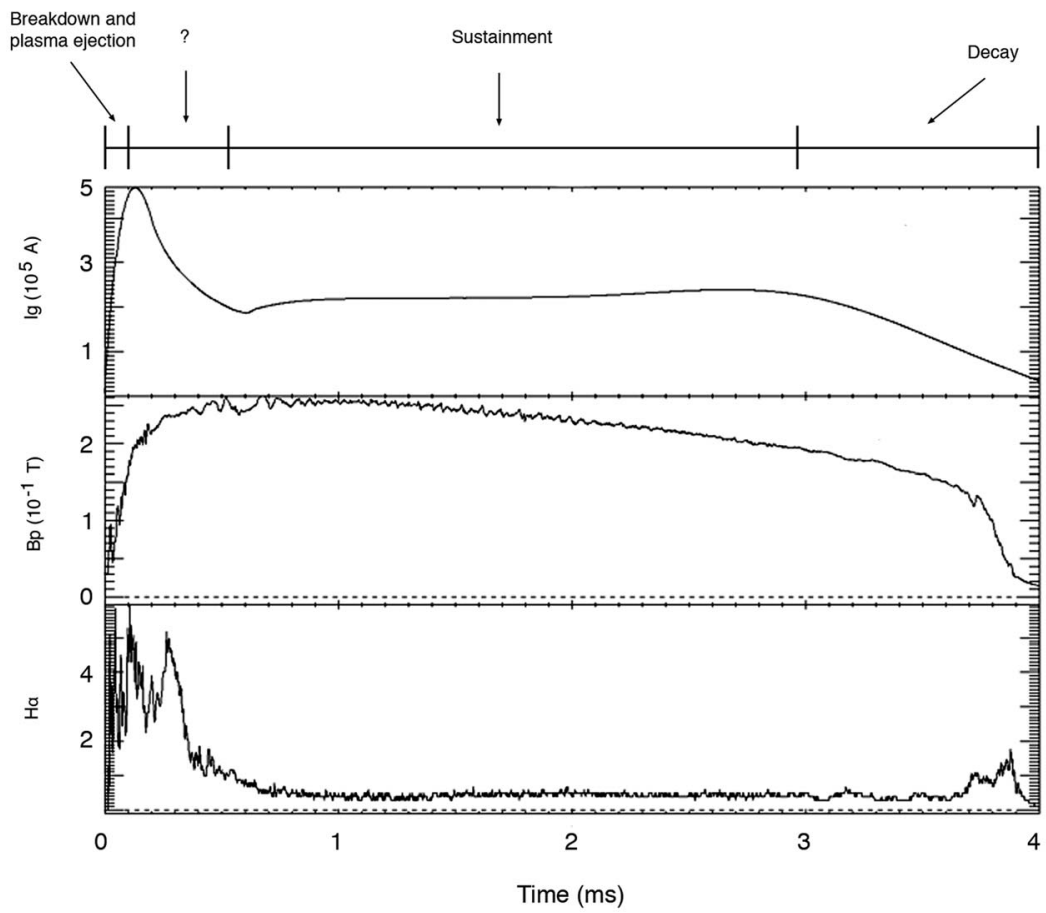

FIG. 2. Top: typical gun current trace at SSPX and the plasma stages identified with the high-speed images. The question mark (?) shown between the breakdown and ejection and the sustainment stages indicates a blind period for the camera, that is, the plasma is either too dim or there is too much plasma in front of the lens to distinguish any features. Middle: edge poloidal magnetic field at midplane. Bottom: line-integrated $H_{\alpha}$ signal from a thin chord perpendicular to the chamber axis at midplane. 


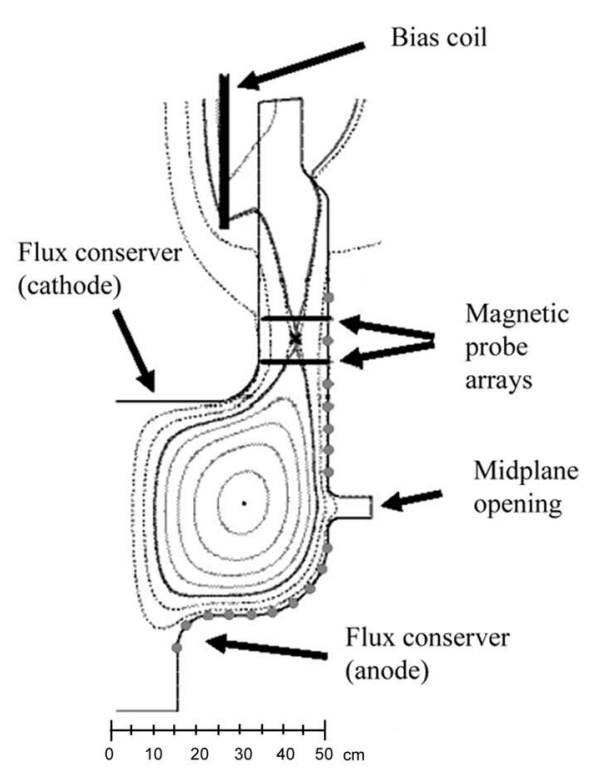

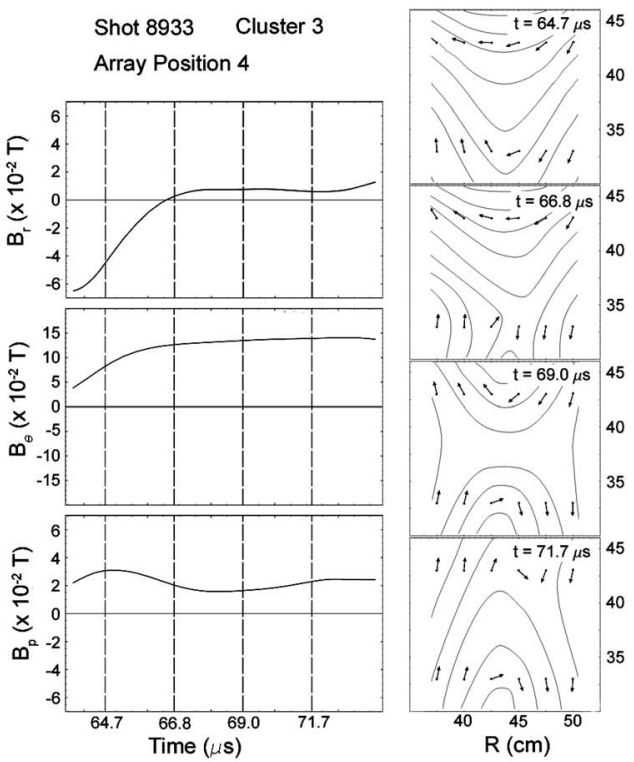

FIG. 3. Two arrays with six $(r, \theta, z)$ clusters of magnetic probes are placed where the $\mathrm{X}$ point are typically expected according to CORSICA reconstructions. One more array was inserted $202^{\circ}$ apart from the pair shown here. The plots in the center show the signal from a single cluster (third from left to right in the lower array) for a short time interval of the first plasma stage. The plots on the right show an $\mathrm{X}$ point (third frame from top to bottom) in a sequence of vector plots that used the $B_{r}$ and $B_{p}$ signals only; contour lines are sketched to show how topology changes near the probes and do not represent precise magnetic-flux values. The dots along the outer wall of the flux conserver represent magnetic probes that measure the toroidal and poloidal fields at the plasma boundary. drifts, the sustainment column images, and measurements done with these images are presented in Sec. IV. A comparison of the measured column radius to numerical reconstructions with a MHD equilibrium code, CORSICA, ${ }^{7}$ is also presented in this section. Images of the decay stage are shown in Sec. V, followed by a discussion of the results in Sec. VI.

\section{EXPERIMENTAL SETUP}

\section{A. High-speed imaging system}

At SSPX, a high-speed camera (Cooke Corporation, model DiCam-Pro) was installed with the capability of obtaining up to two $1280 \times 1024$ pixel images per plasma shot with shutter speeds as fast as 2 ns. The camera was placed outside the vacuum chamber, and optical access to the plasma was achieved with the use of specially designed reentry ports that placed a window through the midplane opening in the flux conserver. A custom relay lens allowed the placement of a camera lens with a field of view that spanned most of the plasma volume. The camera placement and field of view are shown in Fig. 1. The capability to synchronize the images to known signals (e.g., plasma breakdown) allowed coordination of camera timing with other SSPX diagnostics. Camera programming and preparation before every shot were done through a computer that was linked to the camera through an optical fiber to avoid arcing outside the experiment room. Since the computer was connected to the internet and used encryption protocols, the camera was frequently controlled remotely from another computer at Caltech, more than $500 \mathrm{~km}$ away from Livermore.

Image contrast depends not only on the optics used but also on the intensified charged couple device (ICCD) exposure time. The shutter speeds needed to get enough contrast varied depending on the time in the SSPX discharge at which the pictures were taken. During the breakdown and ejection stage (Sec. III) features are bright but move fast (tens of $\mathrm{km} / \mathrm{s}$ ). Shutter speeds needed for this stage ranged between 100 and 200 ns. During the sustainment stage (Sec. IV) fea- tures move considerably slower, but brightness is much lower than the first stage; shutter speeds required ranged between 1 and $4 \mu \mathrm{s}$. For the decay stage (Sec. V) shutter speeds needed varied between $100 \mathrm{~ns}$ and $1 \mu \mathrm{s}$. The limited amount of light entering the camera through the custom optics as well as a low intensifier gain setting (25\% or less) were found to be sufficient in order to avoid ghosting. ${ }^{8}$

\section{B. Injector magnetic probes}

Three arrays of magnetic probes, each with six clusters of induction loops that measured $B_{r}, B_{\theta}$, and $B_{z}$, were installed in the gun injector region (i.e., near the exit of the gun towards the flux conserver region). Two arrays were placed at the same toroidal location, a few centimeters apart as shown in Fig. 3. The third array was placed at approximately the same $z$ location as the other two, but separated by a toroidal angle of $202^{\circ}$. The three arrays were enclosed in boron nitride tubes to provide electrical and thermal isolation to the inductors and were mounted on sliding rails for precision placement or to remove them from the chamber when not in use.

The voltage signal from each inductor was digitized at a maximum frequency of $3 \mathrm{MHz}$ and, with the usual expressions for induction probes, ${ }^{9}$ integrated digitally to obtain the value of the magnetic field at each cluster.

\section{BREAKDOWN AND EJECTION STAGE}

In this stage the plasma typically breaks down in the gun region, outside the camera field of view. Initially, only the bias field with radial and axial components is present in the vacuum chamber and is constant in time. Neutral gas is then puffed into the gun region and the high-voltage switch from the main capacitor bank is closed to initiate breakdown. Breakdown time is defined as $t=0$ for all images. After breakdown, $\mathbf{J} \times \mathbf{B}$ forces rotate and push the plasma into the flux conserver region $\left(F_{\theta}=J_{z} B_{r}-J_{r} B_{z}\right.$ and $F_{z}=J_{r} B_{\theta}-J_{\theta} B_{r}$, with $B_{\theta}$ appearing as soon as current flows through the cath- 


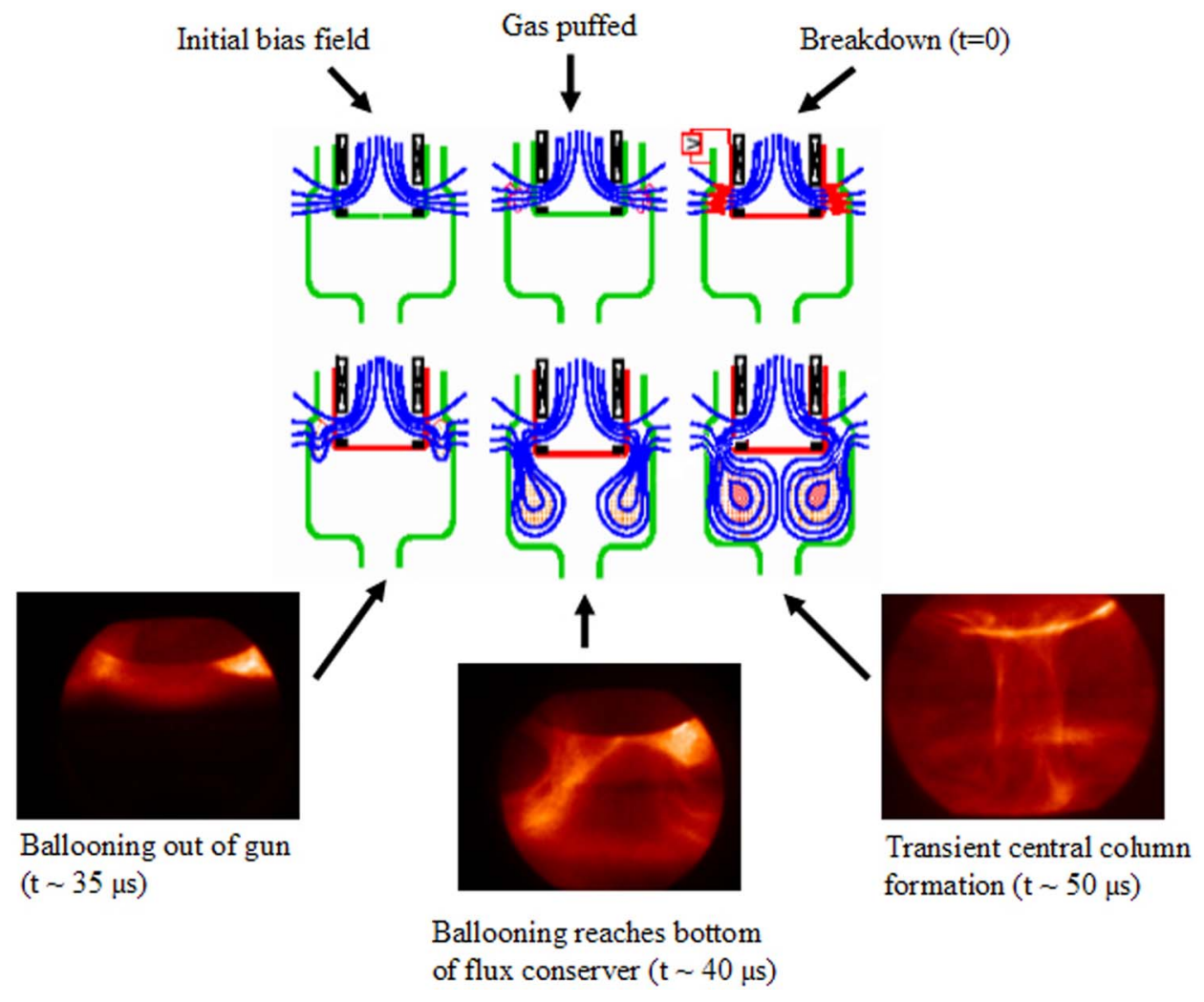

FIG. 4. (Color online) Plasma expansion into the flux conserver during the breakdown and ejection stage.

ode). As the plasma moves into the flux conserver, it drags and deforms the poloidal magnetic field. This process is shown in Fig. 4.

The plasma enters the high-speed camera field of view a few tens of microseconds after breakdown, as seen on the left image in Fig. 5(a). Some structure can be observed [right image in Fig. 5(a)] just a few microseconds later, as the plasma continues traveling along the axis of the gun. The structure typically resembles descending theater curtains due to its undulations. At this stage, the expansion speed of these "curtains" into the flux conserver region is of the order of $50 \mathrm{~km} / \mathrm{s}$. As the plasma continues filling the rest of the flux conserver, the undulations merge to form a more uniform expansion front. This merging is likely caused by the plasma rotating around the axis of the gun.

Magnetic probe arrays inserted in the gun region (see Fig. 3) show that the magnetic field is not ejected symmetrically. These probes, separated $202^{\circ}$ around the chamber axis, also indicate the presence of an $\mathrm{X}$ point after breakdown and during sustainment. Reversal of the radial field in the coaxial gun region indicates closed magnetic-flux surfaces and occurs when the gun current $I_{g}$ is ramped up above the ejection threshold $^{10,11} I_{c r} \sim 15 \phi_{o} / \mu_{o}$, where $\phi_{o}$ is the gun flux and the numerical factor is related to the chamber geometry. When $I_{g}>I_{c r}$, the $\mathrm{X}$ point moves towards the flux conserver. Because no probes are inserted in the flux conserver region, it is not known if the $\mathrm{X}$ point disappears or simply remains out of the gun region. When $I_{g}<I_{c r}$, the $\mathrm{X}$ point moves back into the gun region.

It is conjectured that the magnetic field conforms to the shape of the "theater curtains" shown in Fig. 5(a). However, this relation could not be tested because the magnetic probes were outside the field of view of the camera, but asymmetries in probe signals are qualitatively similar to asymmetries observed in the expansion front of these theater curtains.

The plasma expansion into the flux conserver is inherently three dimensional since the plasma moves radially (inwards), toroidally, and axially as it balloons into the flux conserver. The axial and toroidal parts of the motion describe a "barber pole" trajectory around the inner electrode. An example of this barber pole motion is shown in Fig. 5(b). A bright spot (indicated with an arrow) is identified on the left image. After $5 \mu \mathrm{s}$, the bright spot moved toroidally almost as much as it has traveled along the axis of the gun. Note that some filaments, probably created in the gun region, are visible on the image on the right. A potential mechanism for filament formation has been studied by Ryutov et al. ${ }^{12}$

It should be noted that at this stage the plasma radial displacement cannot be discerned from the high-speed images (which, after all, are two-dimensional projections). However, images such as those shown in Fig. 5(c) taken at later times suggest that at this time the plasma moves much faster helicoidally (describing the barber pole motion) than radially. In the Fig. 5(c) images, the expansion front reaches the flux conserver bottom (about $50 \mathrm{~cm}$ away from the edge of the electrode) faster than it reaches the center of the inner electrode (about $25 \mathrm{~cm}$ away from the edge). During this expansion, a central column becomes more apparent. We refer to this column as the transient central column because it is very short lived, as will be shown below.

Filamentation within the transient central column is sometimes visible. These filaments are probably the evolution of the filaments observed at earlier times, such as those shown in Fig. 5(b). Figure 5(c), for example, shows filaments 

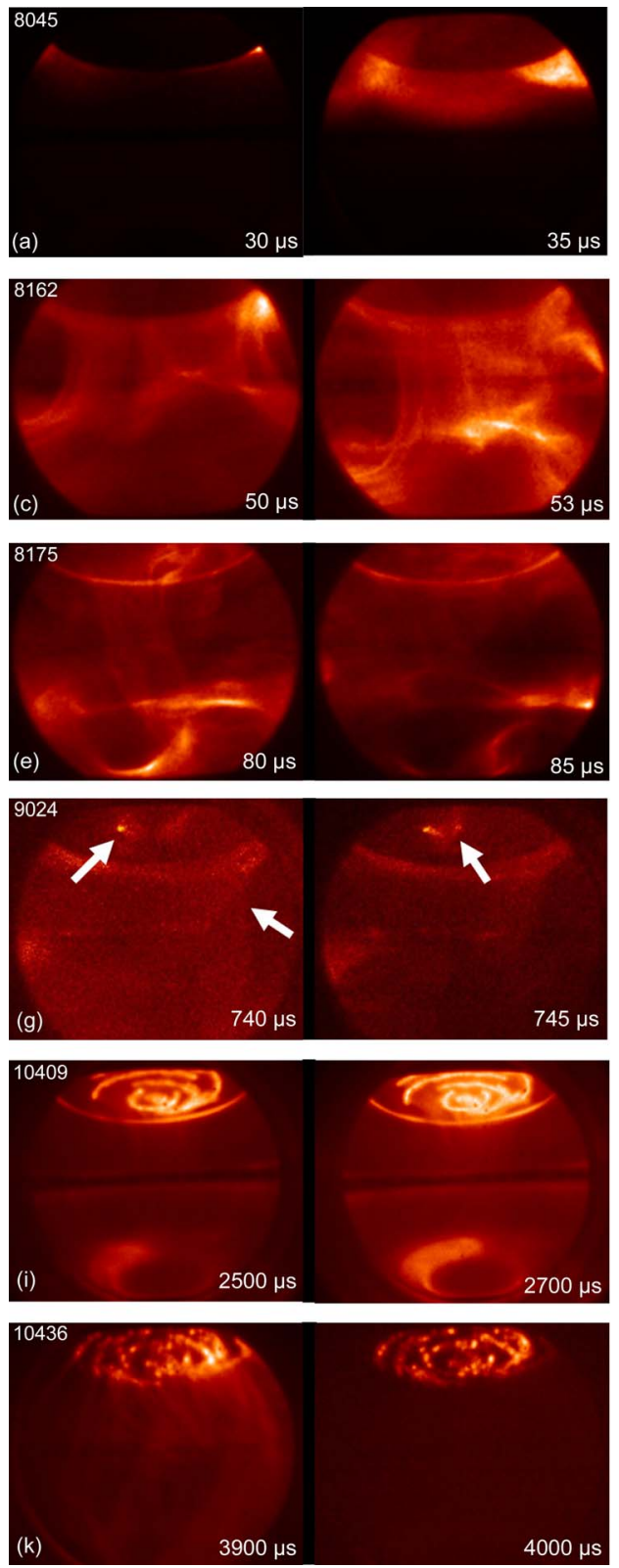
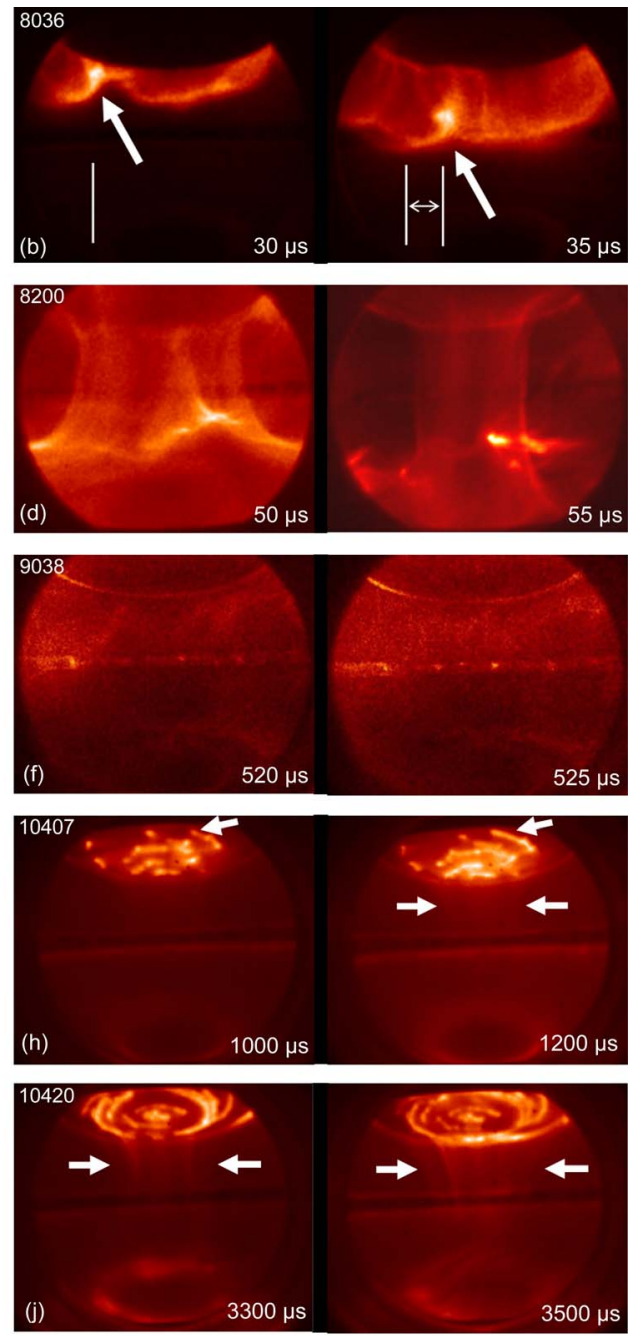

FIG. 5. (Color online) Images from all stages of the SSPX plasma. Hydrogen was used in all shots, except for (e), which used helium. Formation and disappearance of the transient central column are slower with helium, but qualitatively the same as hydrogen. A SSPX shot number is shown on the upper left-hand corner of each image pair. in the left side of both images. The filaments seem to be "stretching" along with the plasma, and localized twisting of those filaments is sometimes observed. As time progresses, the diameter of the central column decreases and the overall emitted light intensity diminishes, as seen in Fig. 5(d).

The transient central column bends rapidly just a few microseconds after forming (hence the name "transient"). Figure 5(e) shows a very faint column on the left image. This column seems to have vanished (right image) $5 \mu$ s later. The current from the gun, however, continues to increase smoothly, as shown in Fig. 6(a), but a voltage spike coincides with the bending process [Fig. 6(b)]. The apparent vanishing of the central column is probably due to the plasma becoming highly ionized so that no light is emitted, or else light is emitted outside the wavelength range of the highspeed camera (for the case of a helium plasma or impurities).

After the bending event, a black-out time occurs during which no imaging is possible because of insufficient light (prolonged exposures yielded blurred images with poor contrast and no structure). This black-out time is indicated with a question mark (?) in Fig. 2. Imaging of the plasma during this time could perhaps be done with other gases (used as tracers and injected at selected times) to maximize the light emitted from the plasma features during small periods of time. However, this approach was not attempted in the present work.

\section{SUSTAINMENT STAGE}

Plasma appears to be nearly fully ionized, and thus emits little light by the time the gun current levels off at approximately $220 \mathrm{kA}$ (at $0.5 \mathrm{~ms}$ in Fig. 2). However, certain characteristic features are nevertheless observed in high-speed images taken during this stage, and two of these features are present throughout sustainment. The first characteristic feature is the formation of a faint central column that is much 


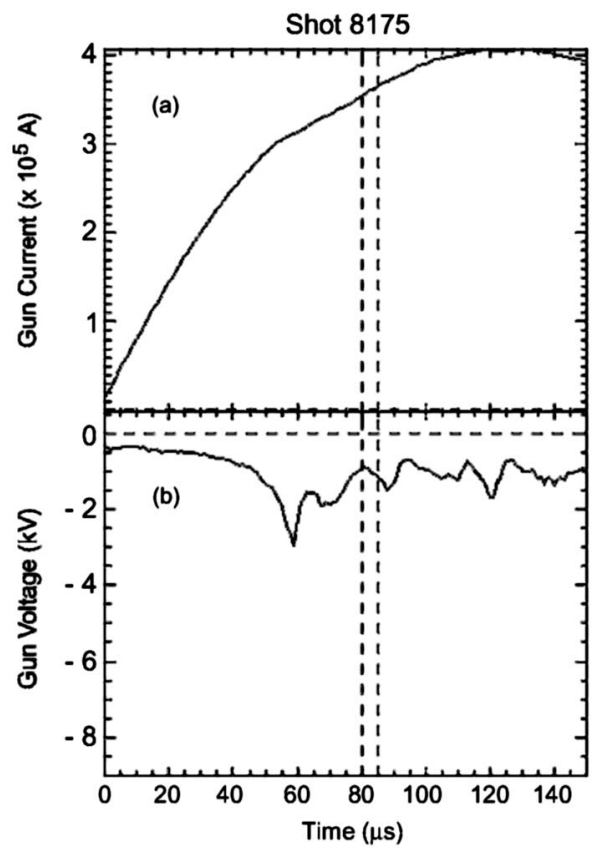

FIG. 6. Gun current and voltage traces for shot 8175 [Fig. 5(e)]. The dotted lines correspond to time of images. The strongest voltage peak (seen here at $60 \mu \mathrm{s})$ corresponds to an expansion similar to that shown in Fig. 5(c).

more stable in time than the column described in the previous section. We call this column the sustainment central column. The second characteristic feature is the spot and pattern formation in the electrodes. The cathode patterns are azimuthally segmented, approximately concentric to the cathode axis, and elongate at discrete radial positions. This pattern elongation appears to be related to plasma drifts. In contrast, the anode patterns, located near the divertor region, are much less well defined than those on the cathode. Measurements of the sustainment column radius are presented in Sec. IV A, and measurements of the cathode pattern elongation are presented in Sec. IV B.

Early in the sustainment stage there are no cathode patterns or indications of a central column. As mentioned earlier, a plasma column (or other features) could be there, but the plasma either does not emit light at all, or it does not emit light in the $280-1000 \mathrm{~nm}$ range ${ }^{13}$ of the high-speed camera. Only some bright spots at the flux conserver midplane can be seen, probably caused by wall recycling and plasma impingement on the posts which hold the flux conserver together. The spots can last tens to hundreds of microseconds, but the position and intensity typically vary in just a few microseconds. Sometimes, there is also a bright glow at the edge of the cathode, as shown in Fig. 5(f).

The bright spots at the midplane posts eventually disappear. Shortly afterwards, spots start to appear on the inner electrode. Figure $5(\mathrm{~g})$ shows two spots that formed at the surface of the electrode. This figure also shows a faint boundary on the left image (arrow above midplane), which could indicate the presence of the sustainment central column or a wide current sheet. Other images taken under similar conditions showed how the spots varied rapidly in intensity (in just a few microseconds), but not in their location on the cathode surface. These spots serve as foot points for the patterns that appear and evolve during the sustainment stage.

The spots grow in number over time scales of tens to hundreds of microseconds. At the same time, patterns start to elongate from these spots. The elongation could be over hundreds of microseconds and extend over centimeters on the cathode surface. It was observed in some images that two or more different patterns could merge or intersect each other, sometimes forming a single and longer pattern (while other patterns in the same shot evolved independently). Figure 5(h) shows an example of the appearance of these patterns during the sustainment stage. Notice how the pattern indicated on the upper part of both images elongated considerably over $200 \mu$ s.

Towards the end of the sustainment stage, the patterns continue to elongate and cover most of the cathode's planar surface, as shown in Fig. 5(i). Also shown in this figure are bright and wide patterns near the divertor opening. These patterns evolve in time just like the patterns on the cathode. However, they seem to vary in intensity and position around the divertor on a much faster time scale (which has not yet been measured) than the cathode patterns. Note the dim glow around the divertor on both images of Fig. 5(h). The patterns near the divertor are very dim (or nonexistent) at the beginning of the sustainment stage, and become brighter as time progresses.

An $\mathrm{X}$ point is also inferred during the sustainment stage from the magnetic measurements in the gun region. If an $n=1$ mode $^{14}$ appears during this stage, it introduces field asymmetries that tend to push the $\mathrm{X}$ point out of the gun, or possibly destroy the outer flux surfaces.

\section{A. Column radius measurements}

The sustainment column radius was measured for a large number of shots with similar conditions using digital contrast enhancement techniques on images of this stage. The measurements are shown in Fig. 7(a). Due to the limitation of the high-speed camera to obtain only two images per shot, it was not possible to describe precisely the change in diameter of the central column throughout a particular shot. However, the ensemble of data indicates a slight column diameter increase (on average) towards the end of the shot.

The best correlation of the column data to SSPX diagnostics was found with the inverse of the poloidal edge magnetic traces. That is, the rate of decrease in poloidal magnetic field at the flux conserver wall correlates well with the rate of increase of the column diameter. A sample of the diagnostic trace for the poloidal field near midplane is shown in Fig. 7(c). Notice that the slope decreases towards the end of the shot, until it sharply decays in less than $200 \mu \mathrm{s}$.

A subset of the shots shown in Fig. 7(b) were selected to compare the measured column diameter with calculations from CORSICA. ${ }^{7}$ This MHD equilibrium code uses the edge magnetic data [like the one shown in Fig. 7(c)] as boundary conditions to reconstruct the magnetic profile. 


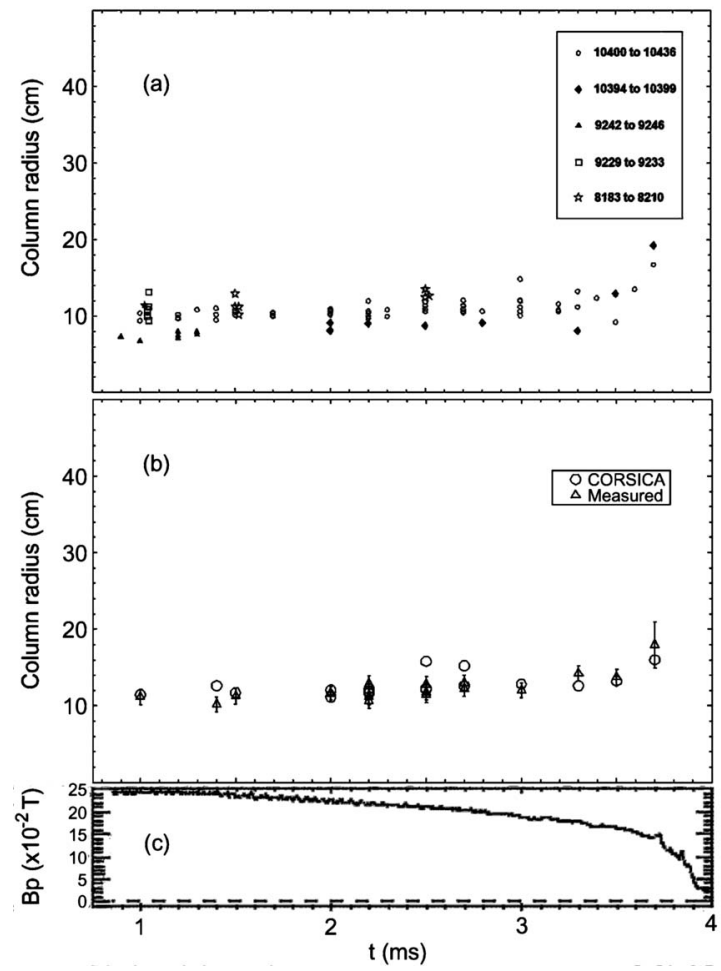

FIG. 7. Column radius during the sustainment and decay stages, and selected radius measurements vs CORSICA. The bottom trace is the typical edge poloidal magnetic field recorded by the probes at midplane.

\section{B. Cathode pattern drifts}

In order to measure features on the cathode, we found it convenient to crop the images to include only the region of interest and then to "stretch" the resulting images. This stretching gives the impression of having the camera at the chamber axis. The pattern elongation was calculated from the initial and final positions of the patterns, as shown in Fig. 8 . The velocity of elongation was calculated from the measured elongation and the time between images of a single shot. These measurements are shown in Fig. 9.

To test the dependence of elongation direction with magnetic field, the bias field was reversed for the same machine operation parameters as those used for the measurements presented above. The measured pattern elongation and velocity remained essentially the same, but the patterns elongated

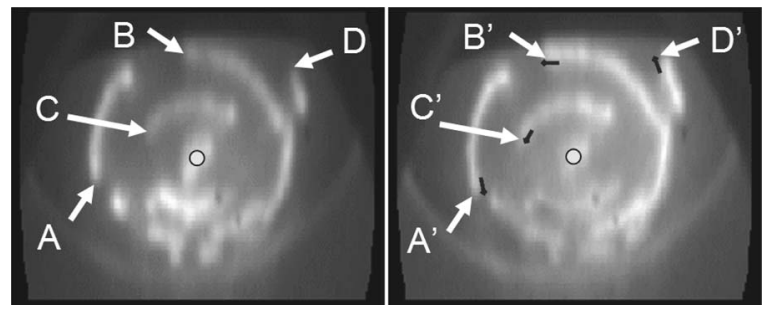

FIG. 8. Example of manual identification of cathode pattern elongation. The center of rotation is identified manually (clear dots), as well as the patterns to be measured (labeled A through D). The small arrows indicate approximate elongation in the counterclockwise direction. Shot 10404, $\Delta t=0.2 \mathrm{~ms}$.

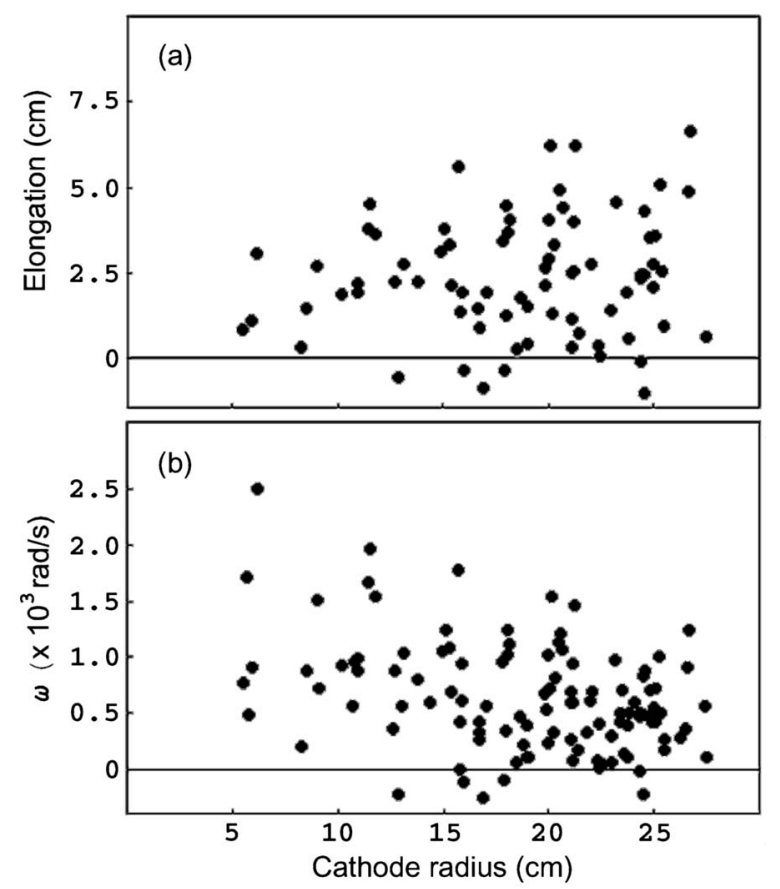

FIG. 9. (a) Cathode pattern elongation vs radius. $\Delta t=0.2 \mathrm{~ms}$. Positive elongation refers to counterclockwise rotation. (b) Angular velocity of patterns vs radius. $\Delta t$ ranges from 0.2 to $0.4 \mathrm{~ms}$.

in the opposite toroidal direction. In both cases, only one side of the pattern (which depended on the sign of $\mathbf{B}$ ) would elongate while the other stayed fixed.

The cause of the pattern elongation is not yet known. No temperature, pressure, electric-field or magnetic-field measurements are feasible at present near the cathode surface, and thus it is not possible to fit the data to theoretical drift models such as drift waves, or $\mathbf{E} \times \mathbf{B}$ drifts, ${ }^{15-17}$ nor has it been possible to explain the variability in the data range.

\section{DECAY STAGE}

At this stage the gun current decreases. Features that were previously dim start to become brighter, thanks to the increase in glowing neutrals. At the beginning of the current ramp down, all the features seen during sustainment remain (i.e., the central column and the cathode and anode patterns). However, changes in the central column do occur. As the current decreases, the central column diameter increases. For example, Fig. 5(j) shows two images taken during the decay stage, with arrows indicating the diameter of the column near midplane. The image on the right shows a column that is brighter and wider. The increase in diameter at the height indicated by the arrows is about $15 \%$ on the second image.

The column diameter continues to increase until at some point the column decomposes into filaments, such as is shown in Fig. 5(k). This decomposition occurred when the gun current had decreased by a factor of 4 or 5 below $I_{c r}$. Both images show that the cathode has patterns similar to those seen at previous times, but these patterns are now segmented, like "strings of pearls." Note that the glow on the cathode shown on the right image could still be caused by 
recycling at the surface since the current flowing into the cathode is still a few kiloamperes. Thus, near the end of the gun current discharge, no more filaments or other features are seen in the images, except for the cathode patterns that fade as the current goes to zero.

\section{DISCUSSION}

The high-speed images, which captured the visible atomic radiation from the plasma and neutrals, revealed unprecedented detail of the SSPX plasma. These images showed the presence of features such as the central plasma columns and filaments in various locations and times throughout the discharge. By exploiting the camera capability to take up to two precisely timed images, it was possible to identify the motion of these filaments and electrode patterns at any given discharge. In some cases, it was also possible to measure the filament displacement and velocity. The camera has access to most of the volume where spheromaks are formed, since it has a wide angle view of the inside of SSPX and the plasma is optically thin for most of a typical discharge. Precise image timing also allowed the synchronization of images with other diagnostics.

Three different stages were identified during the plasma shots according to the distinct plasma features observed. These stages are (i) breakdown and ejection, (ii) sustainment, and (iii) decay. During the first stage, plasma descends from the gun into the flux conserver region in the first hundred microseconds after breakdown, forming a transient plasma column. Capturing impulsive changes of the transient central column proved to be challenging. Although many images were obtained to try to capture this process, none showed more detail than that shown in Fig. 5(e), even when decreasing the time between images to less than $1 \mu \mathrm{s}$.

How the plasma rearranges itself shortly after the transient column bends continues to be a mystery since the gun current flows uninterrupted. It is conjectured that this impulsive bending precedes a reconnection event that leads to flux amplification $^{18}$ via the conversion of toroidal flux (azimuthal to the chamber axis) to poloidal magnetic flux (aligned with the chamber axis). Bellan ${ }^{19}$ proposed a topological constraint of two full turns in a flux rope (or column in our case) for flux to be amplified and helicity to be conserved on the time scale of the reconnection event. This two-turn constraint applied to SSPX is shown in Fig. 10. The high-speed images only show the initial part of this bending and twisting. Highly ionized and very dim plasma prevented imaging of the later part of the column bending and twisting event. If only one turn occurs before reconnection, then the helicity content would increase discontinuously (i.e., a stepwise jump; more details on the geometric interpretation of helicity can be found in Ref. 6). Local magnetic-field measurements would be required to fully test the two-turn hypothesis formulated here.

During the sustainment stage, patterns on the end face of the cathode were observed to elongate in a toroidal-only direction. At the beginning of the sustainment stage only a few cathode spots were observed and served as foot points for the patterns which elongated, on average, with a constant veloc-
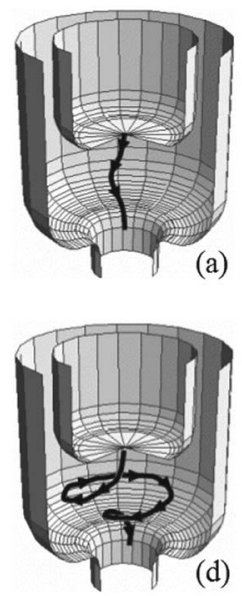

(d)

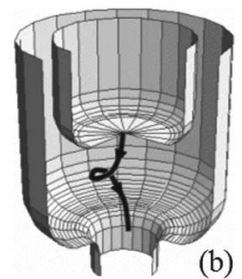

(b)

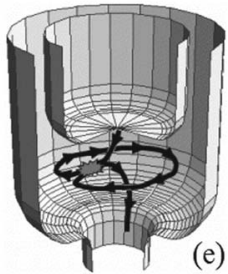

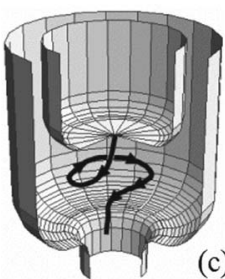

(c)

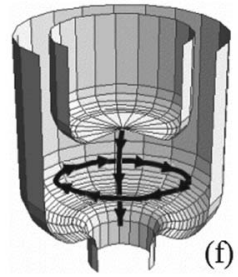

FIG. 10. Bending of the transient central column, and the two-turn constraint for flux amplification. The images on Fig. 5(e) correspond to somewhere between frames (a) and (c). Reconnection occurs in frame (e), resulting in a flux rope linked as in frame (f).

ity. This velocity was measured to be $\sim 0.25 \mathrm{~km} / \mathrm{s}$ in the toroidal direction and depended on the magnetic-field polarity near the cathode.

It is possible that $\mathbf{E} \times \mathbf{B}$ drifts or pressure gradients are responsible for the observed pattern elongation. However, measurements of voltage, magnetic field, and current profile near the cathode surface needed to identify the cause for the pattern drifts, have not been performed, and are the subject of future work. Understanding the cathode patterns could shed light on the formation and evolution of closed (and open) magnetic-flux surfaces, and in the engineering and geometrical considerations for future cathode designs.

During the sustainment stage, information from the highspeed imaging system has also been used to compare the width of the sustainment central column with the CORSICA code. The comparison showed that the numerical reconstructions are in good agreement with the set of measurements presented here. The typical sustainment column radius is about $12 \mathrm{~cm}$, increasing slightly towards the end of the sustainment stage. All the shots used for the comparison had very similar parameters.

At the end of the sustainment stage and beginning of the decay stage, the central column and the cathode patterns are still present. As the gun current decreases, the central column diameter increases and at a current level several times lower than $I_{c r}$, the column breaks up into many filaments. No closed magnetic surfaces are expected after this breakup. The cathode patterns also change, going from the long circular patterns seen during the sustainment stage, to a more segmented version resembling a string of pearls in the decay stage.

An $\mathrm{X}$ point was measured using three magnetic probe arrays placed in two different toroidal locations in the gun region. The $\mathrm{X}$ point was measured for all stages of the plasma discharge, and it could move in and out of the flux conserver depending on gun current levels and the appearance of an $n=1$ mode. The precise shape of the $\mathrm{X}$ point around the chamber is not known. To improve this, more probes in different toroidal locations would be required. 
However, the $\mathrm{X}$ point was inferred to be essentially two dimensional, except on the appearance of an $n=1$ mode, or asymmetric plasma ejection during the first stage.

\section{ACKNOWLEDGMENT}

This work was supported by U.S. Department of Energy.

${ }^{1}$ E. B. Hooper, L. D. Pearlstein, and R. H. Bulmer, Nucl. Fusion 39, 863 (1999).

${ }^{2}$ H. S. McLean, A. Ahmed, D. Buchenauer et al., Rev. Sci. Instrum. 72, 556 (2001)

${ }^{3}$ Z. Wang, G. A. Wurden, C. W. Barnes, C. J. Buchenauer, H. S. McLean, D. N. Hill, E. B. Hooper, R. D. Wood, and S. Woodruff, Rev. Sci. Instrum. 72, 1059 (2001)

${ }^{4}$ Y. Roh, C. W. Domier, and N. C. Luhmann, Jr., Rev. Sci. Instrum. 74, 1518 (2003).

${ }^{5}$ B. W. Stallard, E. B. Hooper, S. Woodruff, R. H. Bulmer, D. N. Hill, H. S. McLean, and R. D. Wood, Phys. Plasmas 10, 2912 (2003).

${ }^{6}$ P. M. Bellan, Spheromaks: A Practical Application of Magnetohydrodynamic Dynamos and Plasma Self-Organization (Imperial College Press, London, 2000), Chap. 3.

${ }^{7}$ A. Tarditi, R. H. Cohen, G. G. Craddock, J. A. Crotinger, G. D. Porter, T. D. Rognlien, A. I. Shestakov, and G. R. Smith, Contrib. Plasma Phys. 36, 132 (1996).
${ }^{8}$ Ghosting is the appearance of artifacts from overexposed features from one image to the next due to intensifier charge depletion. It is a common problem in ICCD plasma imaging.

${ }^{9}$ R. H. Huddleston, S. L. Leonard, and R. H. Loveberg, Plasma Diagnostic Techiques (Academic, New York, 1965), Chap. 3.

${ }^{10}$ C. W. Barnes, J. C. Fernández, I. Henins, H. W. Hoida, T. R. Jarboe, S. O. Knox, G. J. Marklin, and K. F. McKenna, Phys. Fluids 29, 3415 (1986).

${ }^{11}$ S. Woodruff, D. N. Hill, B. W. Stallard, R. Bulmer, B. Cohen, C. T. Holcomb, E. B. Hooper, H. S. McLean, J. Moller, and R. D. Wood, Phys. Rev. Lett. 90, 095001 (2003).

${ }^{12}$ D. D. Ryutov, R. H. Cohen, and D. N. Hill, Plasma Phys. Rep. 29, 605 (2003).

${ }^{13}$ Maximum ICCD response range. The actual response range was reduced depending on the relay lenses used.

${ }^{14}$ D. N. Hill, R. H. Bulmer, B. I. Cohen et al., Proceedings of the 19th IAEA Fusion Energy Conference, Lyon, 2002 (unpublished).

${ }^{15}$ R. J. Goldston and P. H. Rutherford, Introduction to Plasma Physics (IOP, Philadelphia, 2000), pp. 24-26.

${ }^{16}$ R. H. Cohen and D. Ryutov, Phys. Plasmas 6, 1995 (1999).

${ }^{17}$ E. Gravier, F. Brochard, G. Bonhomme, T. Pierre, and J. L. Briançon, Phys. Plasmas 11, 529 (2004).

${ }^{18}$ S. C. Hsu and P. M. Bellan, Phys. Plasmas 12, 032103 (2005).

${ }^{19}$ See EPAPS Document No. E-PHPAEN-12-034512 for P.M. Bellan's Innovative Confinement Workshop oral presentation (Madison, 2004). This document can be reached via a direct link in the online article's HTML references section or via the EPAPS homepage (http://www.aip.org/ pubservs/epaps.html). 